\title{
Rope of Cyclical Helical Surfaces
}

\author{
Tatiana Olejníková \\ Technical University of Košice \\ Faculty of Civil Engineering, Institute of Technology, Economics and Management in Civil Engineering \\ e-mail: tatiana.olejnikova@tuke.sk
}

\begin{abstract}
This paper describes one method for modeling the rope of cyclical helical surfaces as surfaces of technical practice. The cyclical helical surfaces are generated by continuous movement of the circle together with the moving trihedron of the helix along this helix. The rope is composed of equal sets of cyclical surfaces in two layers. In this paper there is described the creation of straight and helical rope and vector functions of the surfaces in the rope are derived. The author visualizes the resulting surfaces in the rope obtained by its parametric approach in the MAPLE program.
\end{abstract}

Key words: cyclical helical surface, helix, vector function of curve and surface, revolution

\section{Introduction}

A rope of cyclical helical surfaces is created of cyclical helical surfaces in two layers (Fig.1). The first layer contains one set and the second layer contains $n$ sets of surfaces screwed about the set of the first layer. Each set of surfaces contains one central surface and $l$ surfaces screwed about this central surface (Fig.3, Fig.4). A particular cyclical helical surface is created by movement of a circle with a radius $r$ (Tab.1) along the helix $s$, where the center of the circle is located on the helix and the circle is located in the normal plane of the helix (Fig.2, Fig.3).

The particular helix will be marked by $s_{\mathrm{kji}}$, where the index $\mathrm{k}=1,2$ marks the layer number, the index $\mathrm{j}=1, \ldots, \mathrm{n}$ marks the set number in the corresponding layer, the index $i=0$ marks the central helix in the corresponding set, $\mathrm{i}=1, \ldots, 1$ marks other helix in this set. Helix $s_{\mathrm{kji}}$ will be described by its vector function with homogeneous coordinates as function of the parameter $v \in\langle 0,2 \pi\rangle$

$$
\mathbf{r}_{\mathrm{kji}}(v)=\left(x_{\mathrm{kji}}(v), y_{\mathrm{kji}}(v), z_{\mathrm{kji}}(v), 1\right)
$$




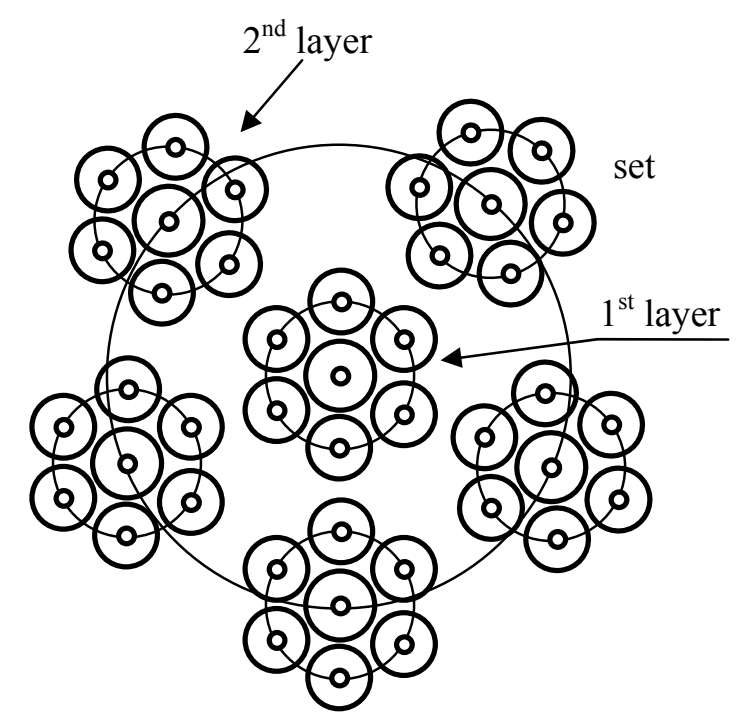

Figure 1: Layout of rope of cyclical helical surfaces

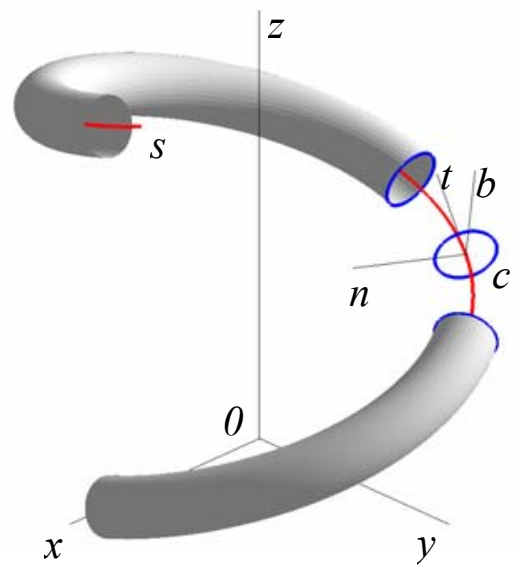

Figure 2

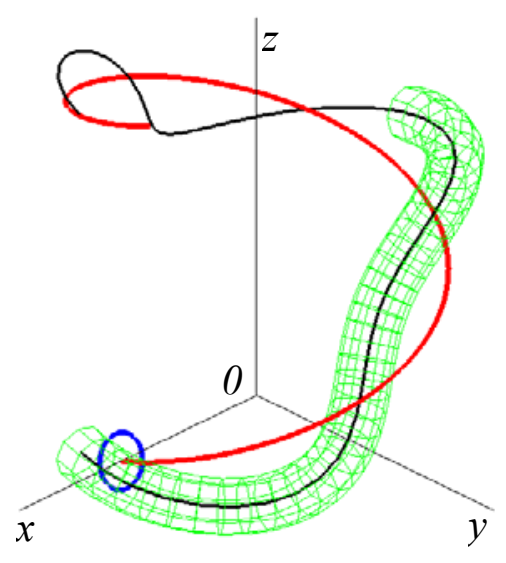

Figure 3

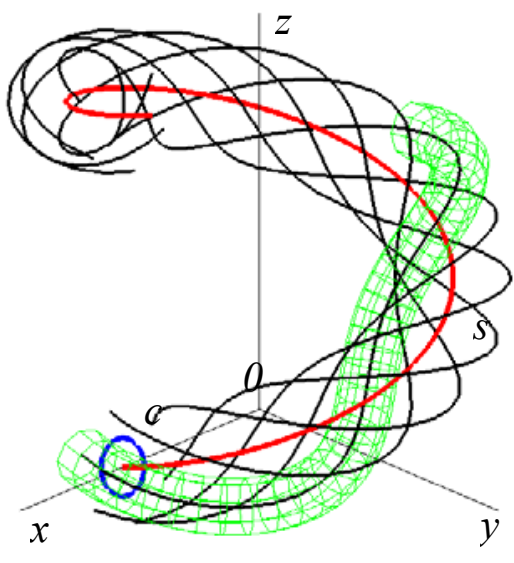

Figure 4

A cyclical helical surface $\Phi_{\mathrm{kji}}$ created by movement of a circle $c$ with a radius $r$ along the helix $s_{\mathrm{kji}}$ will be described by the vector function

$$
\mathbf{P}_{\mathrm{kji}}(u, v)=\mathbf{r}_{\mathrm{kji}}(v)+(r \cos u, r \sin u, 0,1) \cdot \mathbf{M}_{\mathrm{kji}}(v)
$$

where parameter $u \in\langle 0,2 \pi\rangle$ and

$$
\mathbf{M}_{\mathrm{kji}}(v)=\left(\begin{array}{cccc}
{ }_{\mathrm{kji}} n_{x}(v) & { }^{\mathrm{kji}} n_{y}(v) & { }^{\mathrm{kji}} n_{z}(v) & 0 \\
{ }^{\mathrm{kji}} b_{x}(v) & { }^{\mathrm{kji}} b_{y}(v) & { }^{\mathrm{kji}} b_{z}(v) & 0 \\
{ }_{\mathrm{kji}} t_{x}(v) & { }^{\mathrm{kji}} t_{y}(v) & { }^{\mathrm{kji}} t_{z}(v) & 0 \\
0 & 0 & 0 & 1
\end{array}\right)
$$


is a transformation matrix of the trihedron $\left(P,{ }^{\mathrm{kji}} n,{ }^{\mathrm{kji}} b,{ }^{\mathrm{kji}} t\right)$ determined by the basic normal, binormal and tangent of the helix $s_{\mathrm{kji}}$ with unit vectors expressed in (4), (5), (6) into the orthogonal coordinate system $(0, x, y, z)$

$$
\begin{gathered}
{ }^{\mathrm{kji}} \mathbf{t}(v)=\left({ }^{\mathrm{kji}} t_{x}(v),{ }^{\mathrm{kji}} t_{y}(v),{ }^{\mathrm{kji}} t_{z}(v)\right)=\frac{\mathbf{r}_{\mathrm{kji}}^{\prime}(v)}{\left|\mathbf{r}_{\mathrm{kji}}^{\prime}(v)\right|}, \\
{ }^{\mathrm{kji}} \mathbf{b}(v)=\left({ }^{\mathrm{kji}} b_{x}(v),{ }^{\mathrm{kji}} b_{y}(v),{ }^{\mathrm{kji}} b_{z}(v)\right)=\frac{\mathbf{r}_{\mathrm{kji}}^{\prime}(v) \times \mathbf{r}_{\mathrm{kjji}}^{\prime \prime}(v)}{\left|\mathbf{r}_{\mathrm{kji}}^{\prime}(v) \times \mathbf{r}_{\mathrm{kji}}^{\prime \prime}(v)\right|}, \\
{ }^{\mathrm{kji}} \mathbf{n}(v)=\left({ }^{\mathrm{kji}} n_{x}(v),{ }^{\mathrm{kji}} n_{y}(v),{ }^{\mathrm{kji}} n_{z}(v)\right)={ }^{\mathrm{kji}} \mathbf{b}(v) \times{ }^{\mathrm{kji}} \mathbf{t}(v) .
\end{gathered}
$$

Table 1: Distribution of the cyclical helical surfaces in the rope with a radius of circle $c$

\begin{tabular}{|c|c|c|c|c|c|}
\hline Layer $\mathrm{k}$ & Set $\mathrm{j}$ & Surface $\Phi_{\mathrm{kj} 0}$ & $r$ & Surface $\Phi_{\mathrm{kji}}$ & $r$ \\
\hline 1 & 1 & $\Phi_{110}$ & $r_{1}$ & $\Phi_{111}, \Phi_{112}, \ldots \Phi_{111}$ & $r_{2}$ \\
\hline 2 & 1 & $\Phi_{210}$ & $r_{3}$ & $\Phi_{211}, \Phi_{212}, \ldots \Phi_{211}$ & $r_{4}$ \\
\hline 2 & 2 & $\Phi_{220}$ & $r_{3}$ & $\Phi_{221}, \Phi_{222}, \ldots \Phi_{221}$ & $r_{4}$ \\
\hline 2 & 3 & $\Phi_{230}$ & $r_{3}$ & $\Phi_{231}, \Phi_{232}, \ldots \Phi_{231}$ & $r_{4}$ \\
\hline 2 & $\vdots$ & $\vdots$ & $\vdots$ & $\vdots$ & $\vdots$ \\
\hline 2 & $n$ & $\Phi_{2 \mathrm{n} 0}$ & $r_{3}$ & $\Phi_{2 \mathrm{n} 1}, \Phi_{2 \mathrm{n} 2}, \ldots \Phi_{2 \mathrm{nl}}$ & $r_{4}$ \\
\hline
\end{tabular}

\section{Straight rope of cyclical helical surfaces}

A straight rope of cyclical helical surfaces is created in such a way that the helix $s_{110}$ is straight line identical with the coordinate axis $z$ of the orthogonal coordinate system $(0, x, y, z)$. Its vector function is

$$
\mathbf{r}_{110}(v)=(0,0, v, 1)
$$

The helix $s_{11 \mathrm{i}}$, for $\mathrm{i}=1, \ldots, 1$ is created by screwing of the point $\left(d_{3} \cos i \alpha, d_{3} \sin i \alpha, 0,1\right)$ located in the coordinate plane $x y$ of the coordinate system $(0, x, y, z)$ in the distance $d_{3}$ about the central helix $s_{110}$. Then the vector function of the helix $s_{11 \mathrm{i}}$ will be

$$
\mathbf{r}_{11 \mathrm{i}}(v)=\left(d_{3} \cos i \alpha, d_{3} \sin i \alpha, 0,1\right) \cdot \mathbf{T}\left(m_{1}, s g_{1}\right),
$$

where $v \in\langle 0,2 \pi\rangle$, angle $\alpha=\frac{2 \pi}{1}$, matrix 


$$
\mathbf{T}(m, s g)=\left(\begin{array}{cccc}
\cos m v & s g \sin m v & 0 & 0 \\
-s g \sin m v & \cos m v & 0 & 0 \\
0 & 0 & 1 & 0 \\
0 & 0 & 0 & 1
\end{array}\right)
$$

expresses revolution about the coordinate axis $z$ with an angular velocity determined by the parameter $m v$ and the parameter $s g=+1$ determines the right-handed and $s g=-1$ lefthanded movement.

The helix $s_{2 \mathrm{j} 0}$, for $\mathrm{j}=1, \ldots, \mathrm{n}$ is created by screwing of the point $\left(d_{2} \cos \mathrm{j} \beta, d_{2} \sin \mathrm{j} \beta, 0,1\right)$ in the normal plane of the helix $s_{110}$ in the distance $d_{2}$ about the tangent of the helix $s_{110}$. Then vector function of the helix $s_{2 \mathrm{j} 0}$ will be

$$
\mathbf{r}_{2 \mathrm{j} 0}(v)=\left(d_{2} \cos \mathrm{j} \beta, d_{2} \sin \mathrm{j} \beta, 0,1\right) . \mathbf{T}\left(m_{2}, s g_{2}\right),
$$

where angle $\beta=\frac{2 \pi}{\mathrm{n}}$.

The helix $s_{2 \mathrm{ji}}$, for $\mathrm{j}=1, \ldots, \mathrm{n}, \quad \mathrm{i}=1, \ldots, 1$ is created by screwing of the point $\left(d_{3} \operatorname{cosi\alpha }, d_{3} \sin i \alpha, 0,1\right)$ in the normal plane of the helix $s_{2 \mathrm{j} 0}$ in the distance $d_{3}$ about the tangent of the helix $s_{2 \mathrm{j} 0}$. Then the vector function of this helix $s_{2 \mathrm{ji}}$ will be

$$
\mathbf{r}_{2 \mathrm{ji}}(v)=\mathbf{r}_{2 \mathrm{j} 0}(v)+\left(d_{3} \cos \mathrm{i} \alpha, d_{3} \sin \mathrm{i} \alpha, 0,1\right) \cdot \mathbf{T}\left(m_{3}, s g_{3}\right) \cdot \mathbf{M}_{2 \mathrm{j} 0}(v),
$$

where angle $\alpha=\frac{2 \pi}{1}$.

In Fig. 5 d) there is displayed the straight rope of cyclical helical surfaces determined by the parameters $m_{1}=1, m_{2}=1, m_{3}=2, s g_{1}=s g_{2}=s g_{3}=s g_{4}=+1$ (all surfaces are right-handed). In case a) there is displayed one set of the surfaces of the first layer, in case b) there is displayed one set of the first layer and one set of the second layer, in case c) there are displayed surfaces $\Phi_{110}, \Phi_{210}, \Phi_{220}, \Phi_{230}, \Phi_{240}$.

In Fig. 6 there is the changed parameter $m_{2}$ of the same straight rope as in Fig.5 and another parameters are the same. In Fig.7 there are ropes with changed parameters given under the corresponding picture. 


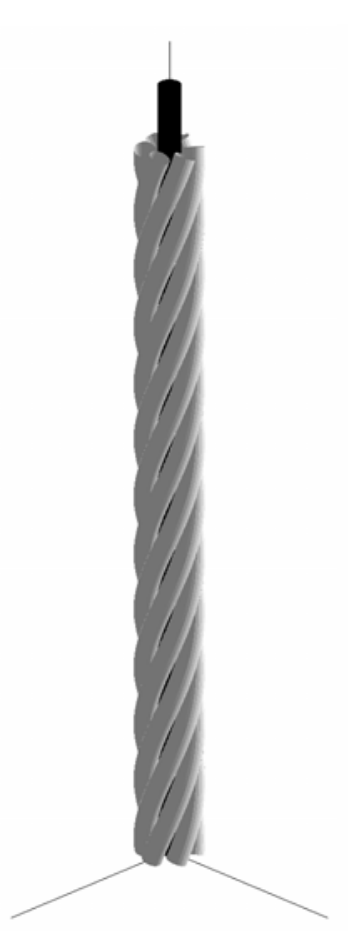

a)

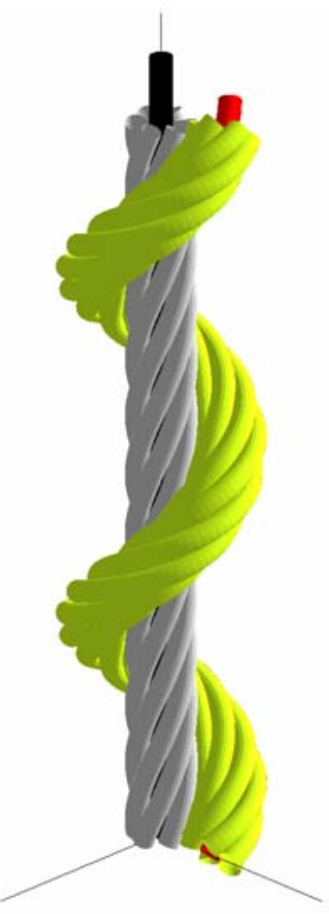

b)

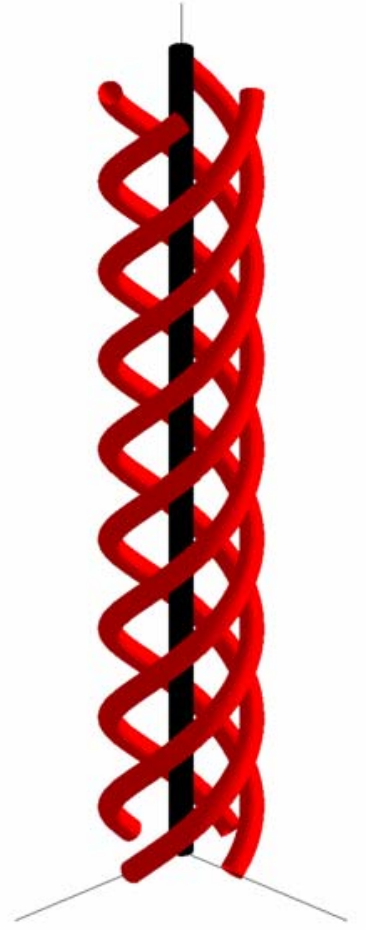

c)

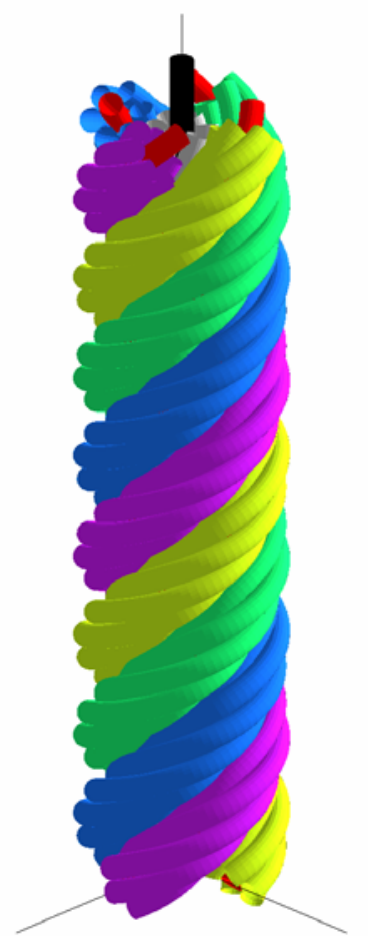

d)

Figure 5: The straight rope of the right-handed cyclical helical surfaces with $n=4, l=6$

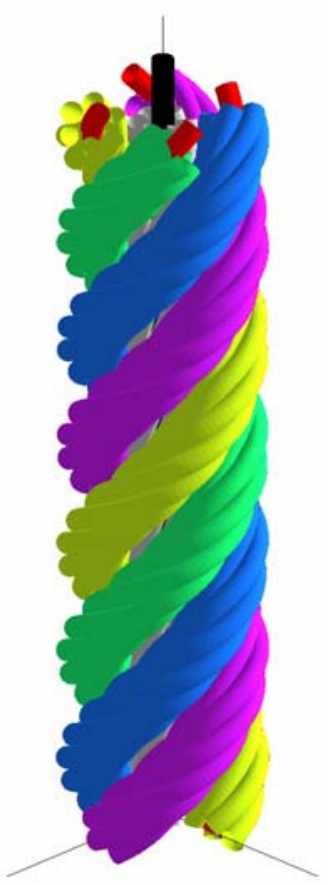

$m_{2}=0.8$
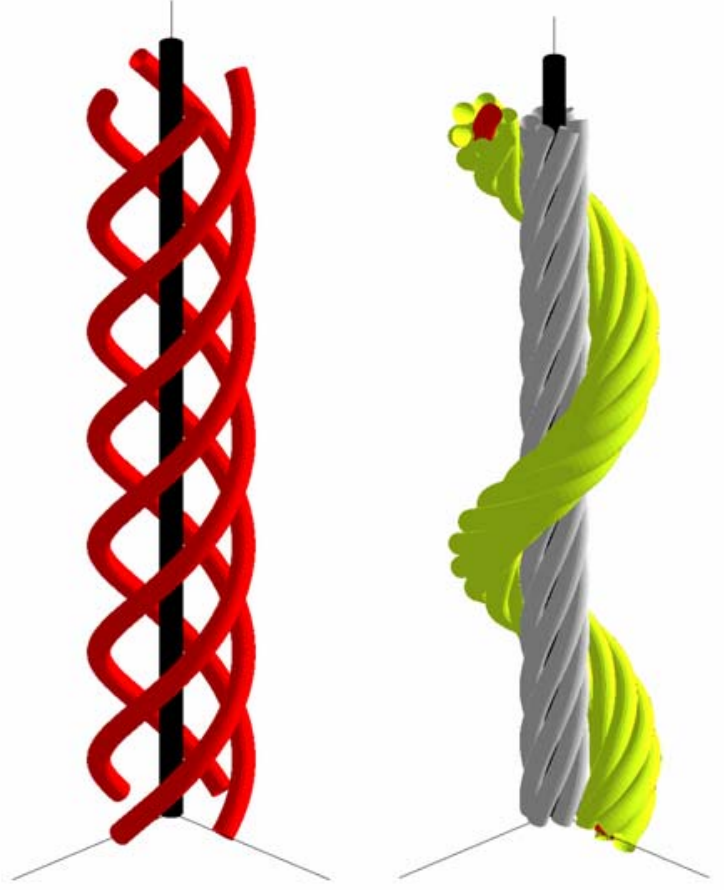

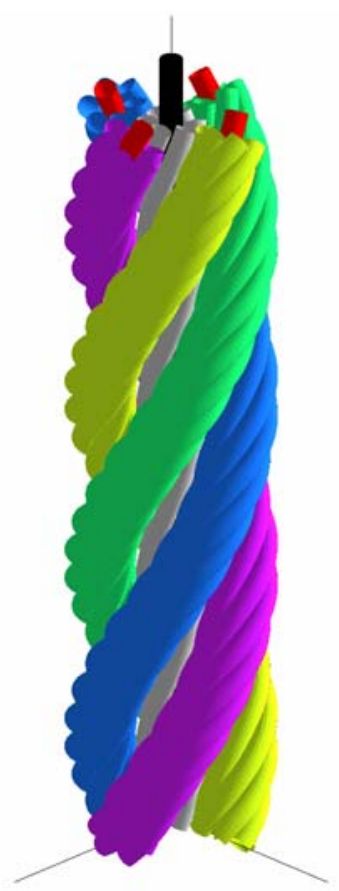

$m_{2}=0.5$

Figure 6: The straight rope of the right-handed cyclical helical surfaces with $n=4,1=6$ 

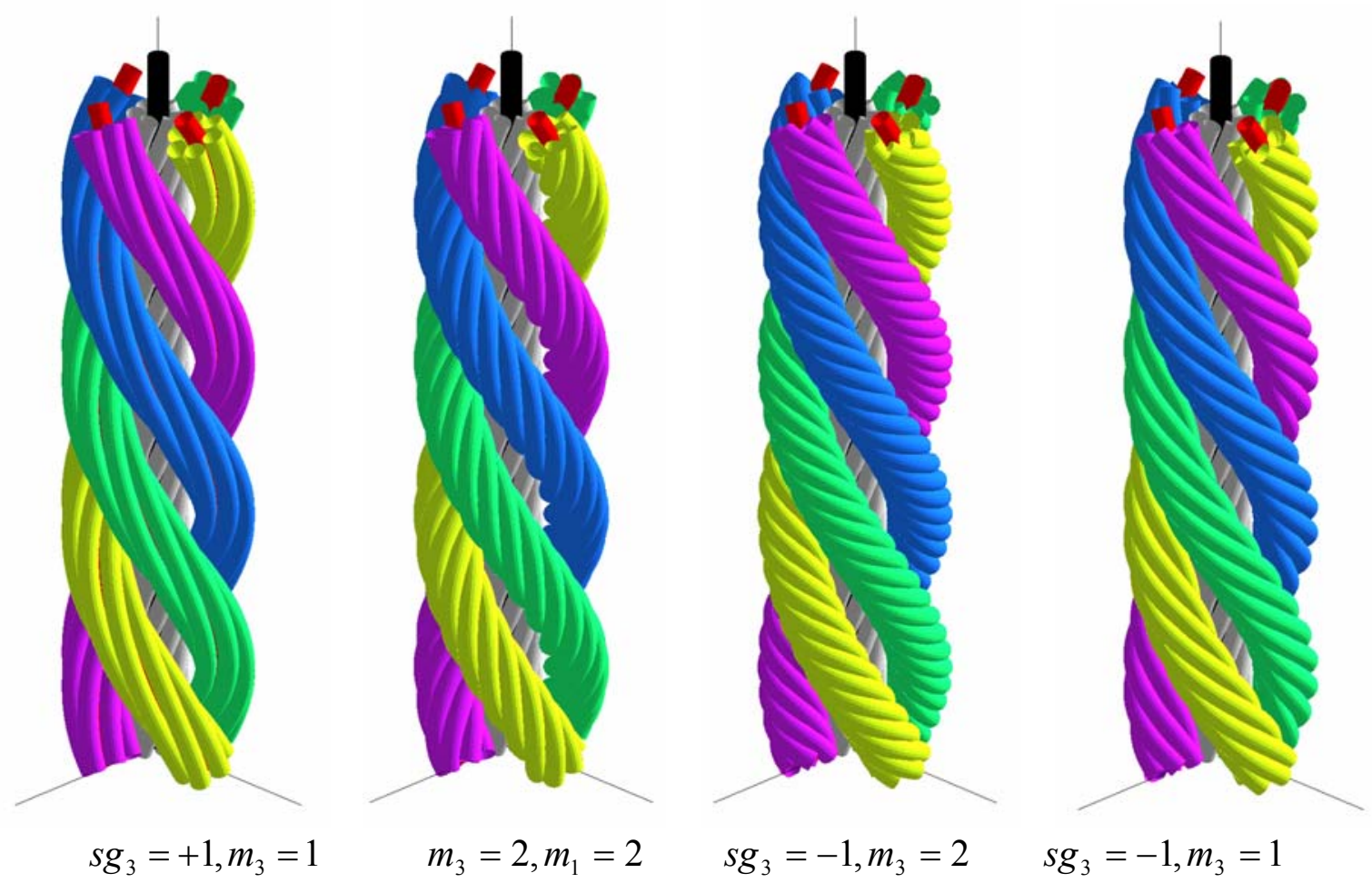

Figure 7: The straight rope of the cyclical helical surfaces with $n=4,1=6$
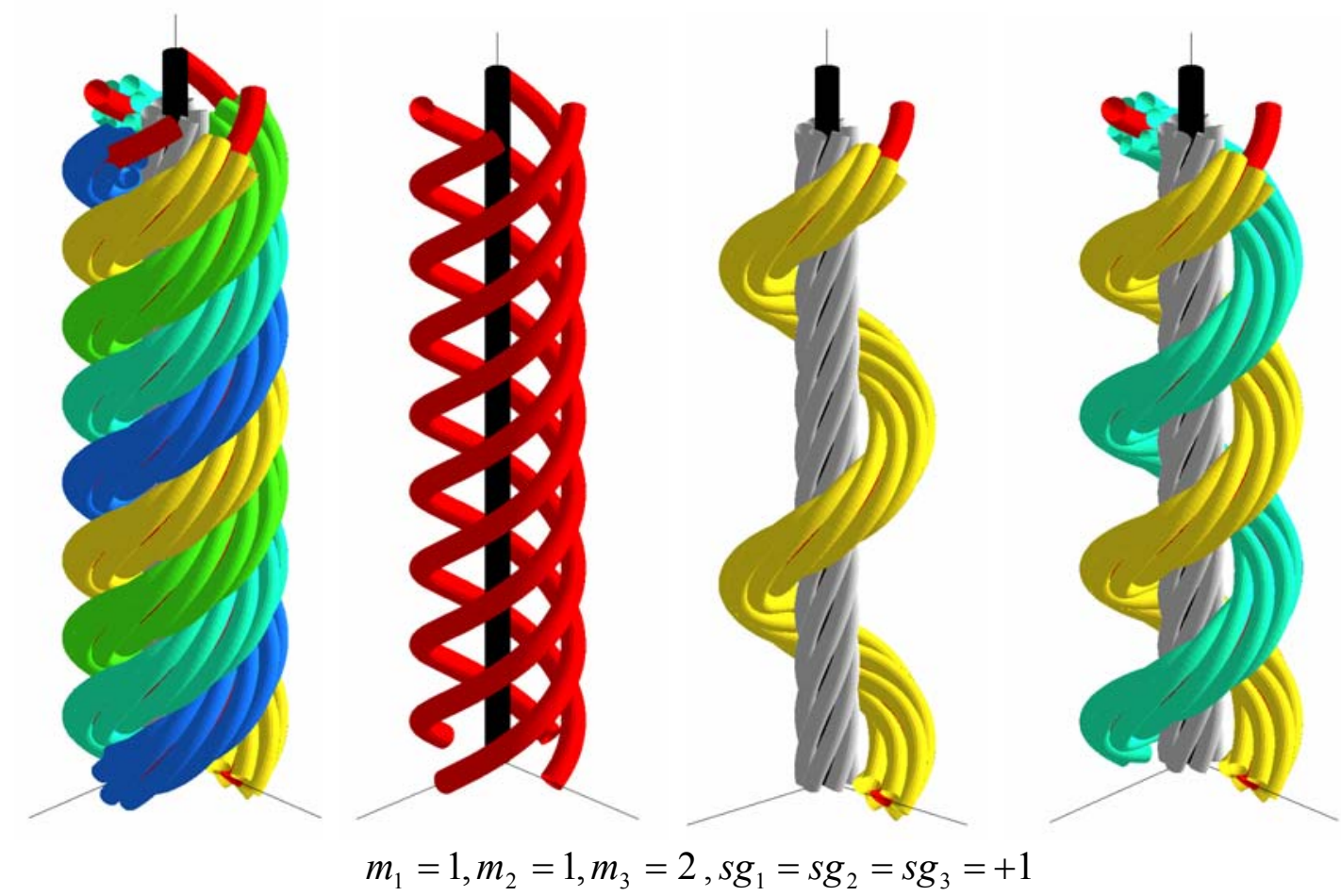

Figure 8: The straight rope of the cyclical helical surfaces with $n=4,1=6$ 


\section{Helical rope of cyclical helical surfaces}

A helical rope of cyclical helical surfaces is created in such a way that the first helix $s_{110}$ is created by screwing of the point $\left(d_{1}, 0,0,1\right)$ about the coordinate axis $z$ of the coordinate system $(0, x, y, z)$ with the reduced pitch $v_{0}$. Its vector function is

$$
\mathbf{r}_{110}(v)=\left(d_{1} \cos v, s g_{1} d_{1} \sin v, v_{0} v, 1\right), v \in\langle 0,2 \pi\rangle,
$$

where the parameter $s g_{1}=+1$ determines a right-handed and $s g_{1}=-1$ left-handed helix.

The helix $s_{11 \mathrm{i}}$, for $\mathrm{i}=1, \ldots, 1$ is created by screwing of the point $\left(d_{3} \operatorname{cosi\alpha }, d_{3} \sin i \alpha, 0,1\right)$ located in the normal plane of the helix $s_{110}$ in the distance $d_{3}$ about the central helix $s_{110}$. Then the vector function of the helix $s_{11 \mathrm{i}}$ will be

$$
\mathbf{r}_{11 \mathrm{i}}(v)=\mathbf{r}_{110}(v)+\left(d_{3} \cos \mathrm{i} \alpha, d_{3} \sin \mathrm{i} \alpha, 0,1\right) \cdot \mathbf{T}\left(m_{1}, s g_{2}\right) \cdot \mathbf{M}_{110}(v),
$$

Where $\alpha=\frac{2 \pi}{1}$, the parameter $m_{1} v$ determines an angular velocity and $s g_{2}$ determines the right-handed or left-handed movement. Matrices $\mathbf{M}$ and $\mathbf{T}$ are in (3), (9).

The helix $s_{2 \mathrm{j} 0}$, for $\mathrm{j}=1, \ldots, \mathrm{n}$ is created by screwing of the point $\left(d_{2} \cos \mathrm{j} \beta, d_{2} \sin \mathrm{j} \beta, 0,1\right)$ in the normal plane of the helix $s_{110}$ in the distance $d_{2}$ about the tangent of the helix $s_{110}$. Then the vector function of the helix $s_{2 \mathrm{j} 0}$ will be

$$
\mathbf{r}_{2 \mathrm{j} 0}(v)=\mathbf{r}_{110}(v)+\left(d_{2} \cos \mathrm{j} \beta, d_{2} \sin \mathrm{j} \beta, 0,1\right) \cdot \mathbf{T}\left(m_{2}, s g_{3}\right) \cdot \mathbf{M}_{110}(v),
$$

where $\beta=\frac{2 \pi}{\mathrm{n}}$, the parameter $m_{2} v$ determines an angular velocity and $s g_{3}$ determines the right-handed or left-handed movement.

The helix $s_{2 \mathrm{ji}}$, for $\mathrm{j}=1, \ldots, \mathrm{n}, \mathrm{i}=1, \ldots, 1$ is created by screwing of the point $\left(d_{3} \operatorname{cosi\alpha }, d_{3} \sin i \alpha, 0,1\right)$ in the normal plane of the helix $s_{2 \mathrm{j} 0}$ in the distance $d_{3}$ about the tangent of the helix $s_{2 \mathrm{j} 0}$. Then the vector function of this helix $s_{2 \mathrm{ji}}$ will be

$$
\mathbf{r}_{2 \mathrm{ji}}(v)=\mathbf{r}_{2 \mathrm{j} 0}(v)+\left(d_{3} \operatorname{cosi} \alpha, d_{3} \sin \mathrm{i} \alpha, 0,1\right) \cdot \mathbf{T}\left(m_{3}, s g_{4}\right) \cdot \mathbf{M}_{2 \mathrm{j} 0}(v),
$$

where $\alpha=\frac{2 \pi}{1}$, the parameter $m_{3} v$ determines an angular velocity and $s g_{4}$ determines the right-handed or left-handed movement. 


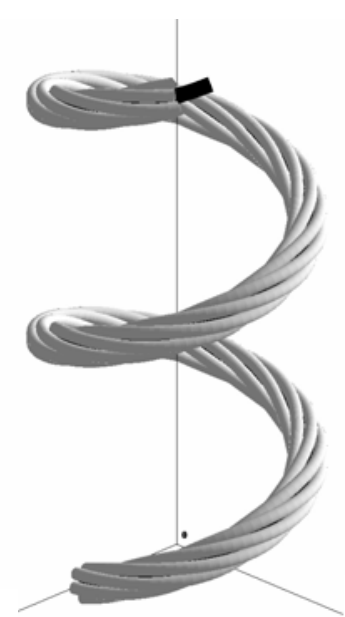

a)

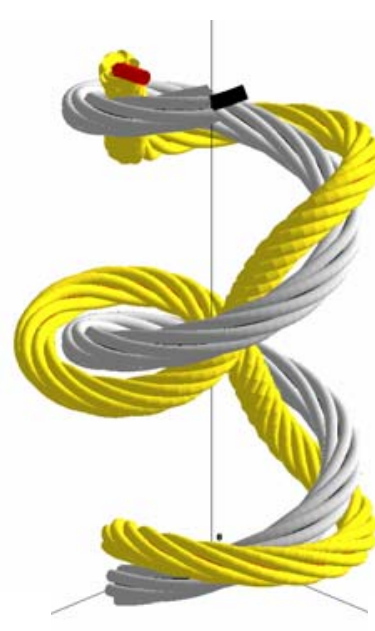

b)

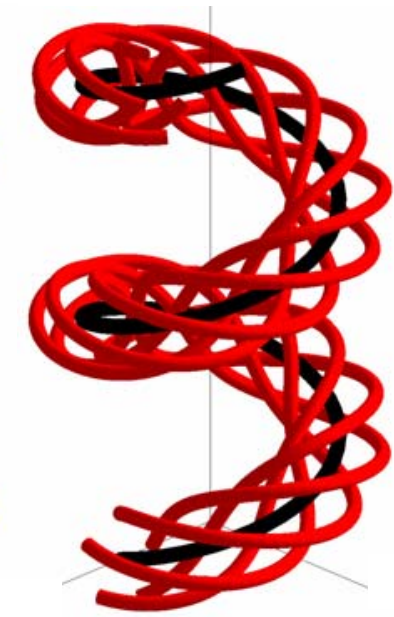

c)

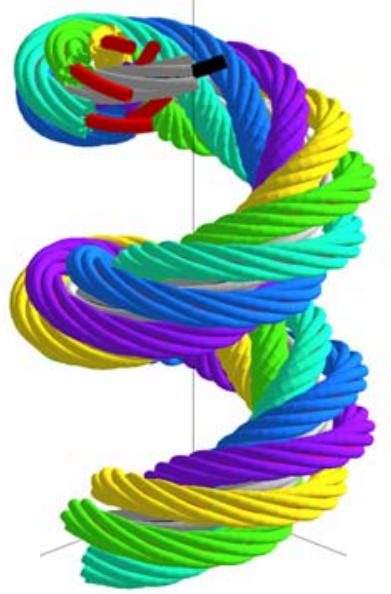

d)

Figure 9: The helical rope of the right-handed cyclical helical surfaces with $n=5,1=6$

In Fig.9 d) there is displayed helical the rope of the cyclical helical surfaces determined by the parameters $m_{1}=2, m_{2}=2.5, m_{3}=4, \quad s g_{1}=s g_{2}=s g_{3}=s g_{4}=+1 \quad$ (all surfaces are righthanded). In case a) there is displayed one set of the surfaces of the first layer, in case b) there is displayed one set of the first layer and one set of the second layer, in case c) there are displayed surfaces $\Phi_{110}, \Phi_{210}, \Phi_{220}, \Phi_{230}, \Phi_{240}, \Phi_{250}$.

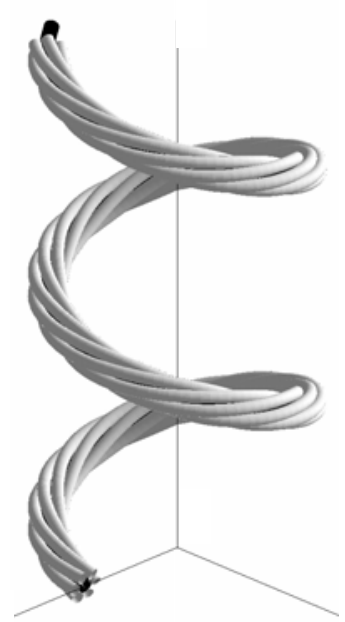

a)

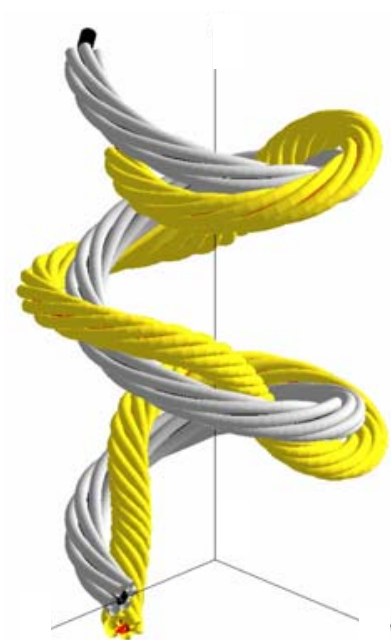

b)

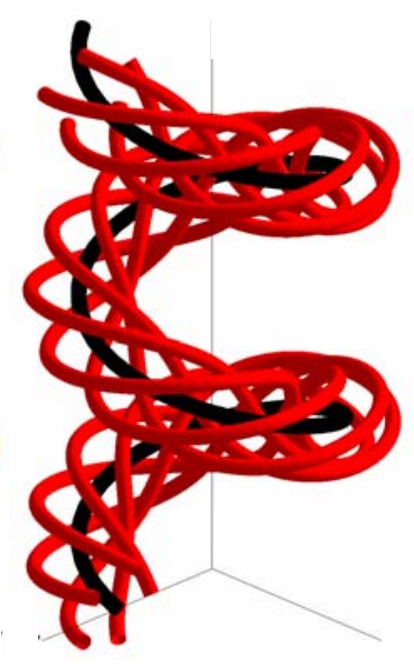

c)

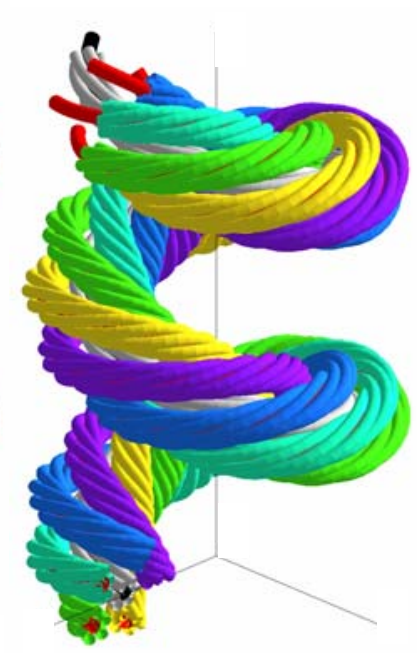

d)

Figure 10: The helical rope of the left-handed cyclical helical surfaces with $n=5,1=6$

In Fig.10 d) there is displayed the helical rope with the parameters $m_{1}=2, m_{2}=2.5, m_{3}=4$, $s g_{1}=s g_{2}=s g_{3}=s g_{4}=-1$. (all surfaces are left-handed). In case a) there is displayed one set of the surfaces of the first layer, in case b) there is displayed one set of the first layer and one set of the second layer, in case c) there are displayed surfaces $\Phi_{110}, \Phi_{210}, \Phi_{220}, \Phi_{230}, \Phi_{240}$, $\Phi_{250}$. 


\section{Conclusion}

A straight rope of the cyclical helical surfaces can be used as a surface around which helical rope screwes as its following layer.

In Fig. 11 there is displayed one straight rope and one or two pitches of the helical rope.
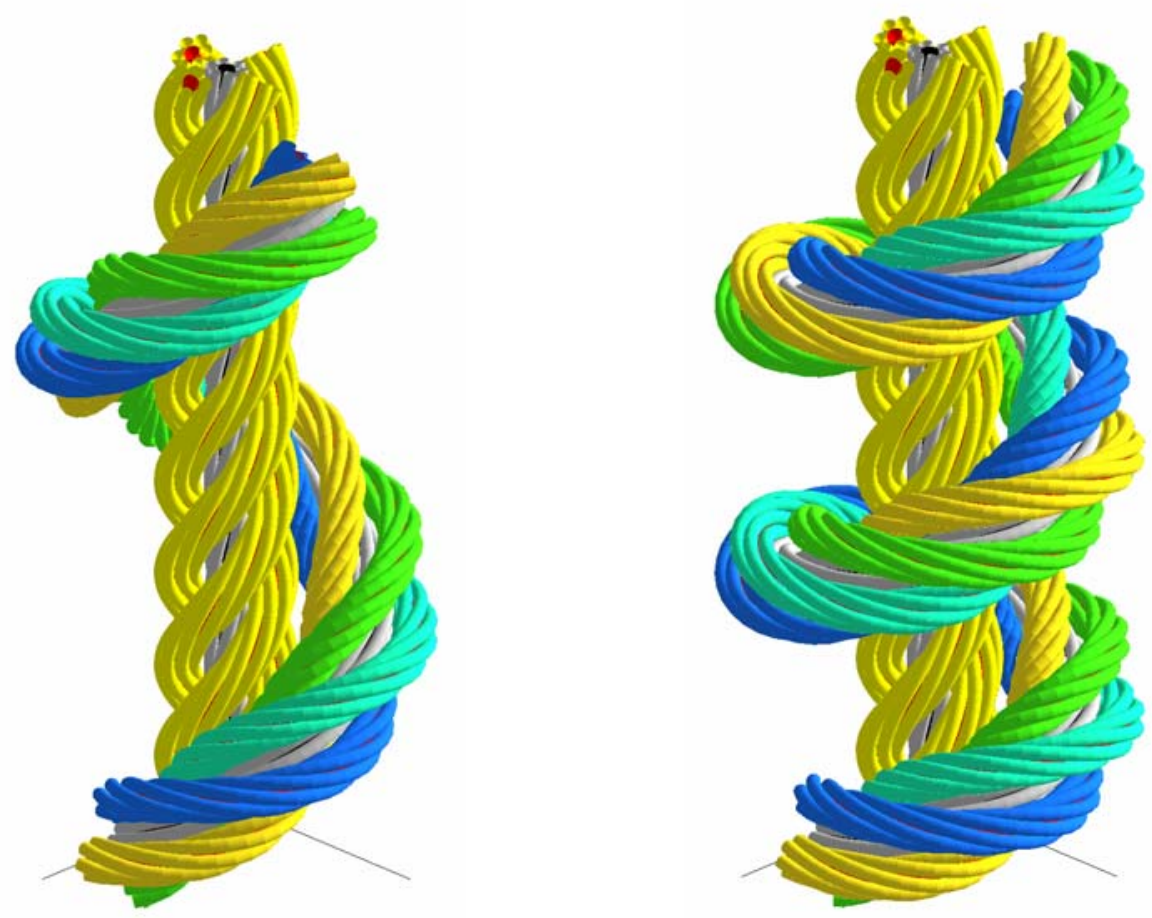

Figure 11: Straight and helical ropes of cyclical helical surfaces with $n=4,1=6$

Vector functions of particular surfaces in the rope of surfaces are created by using parameters by which we can simulate different types of ropes.

As the conclusion, it can be summarized that the presented rope of cyclical helical surfaces serves as an endlessly rich source of inspiration for design purposes. Their unusually complex forms are obtained in a relatively simple way of composite spatial transformations.

\section{Acknowledgements}

This work was supported by VEGA 1/0321/12 „Theoretical and experimental analysis of adaptive cable and tensegrity systems under static and dynamic stress considering the effect of wind and seismic“.

\section{References}

[1] Budinský, B. \& Kepr, B. (1970). Basic of Differetial Geometry with Technical Applications. Praha, SNTL - Publishers of Technical Literature 
[2] Granát, L. \& Sechovský, H. (1980). Computer Graphics. Praha, SNTL - Publishers of Technical Literature

[3] Medek, V. \& Zámožík, J. (1974). Constructive geometry for technics. Bratislava, ALFA Publishers of Technical and Economical Literature

[4] Olejníková, T. (2007). Cyclical Surfaces Created by a Conical Helix. KoG, ScientificProfessional Journal of Croatian Society for Geometry and Graphics. Vol. 11, pp. 33-38

[5] Olejníková, T. (2009). Two-Revolutional Cyclical Surface. Acta Polytechnica Hungarica, Journal of Applied Sciences. Vol.6, (Issue 4), pp. 101-110

[6] Olejníková, T. (2009). Helical two-revolutional linear surface. Zeszyty naukove. Vol.2, (Issue 1), pp. 116-123

[7] Olejníková, T. (2006). Zložené cyklické plochy. G-Slovenský časopis pre geometriu a grafiku. Vol. 3, (Issue 6), pp. 21-28

[8] Olejníková, T. (2007). Composed Cyclical Surfaces. Transactions of the universities of Košice, Vol.3, pp. $54-60$

[9] Olejníková, T. (2008). Two and three-revolution cyclical Surfaces. Communications, Scientific Letters of the University of Žilina, Vol. 3, pp. 72-76

[10] Olejníková, T. (2010). Three-Helical Cyclical Surfaces. G, Slovenský časopis pre geometriu a grafiku. Vol. 7, (Issue 13), pp. 37-44 